\title{
Thesis
}

\section{Routine as resource for the design of learning systems}

\author{
Scott Davidoff \\ Carnegie Mellon University \\ Committee: \\ Anind K. Dey \\ Carnegie Mellon University \\ John Zimmerman \\ Carnegie Mellon University \\ Scott E. Hudson \\ Carnegie Mellon University \\ Gregory D. Abowd \\ Georgia Institute of Technology
}

Keywords: Machine learning, data mining, routine, intelligent system, visualization, GPS, mobile phones, families

The ubiquitous computation that underlies ambient intelligence offers an unprecedented framework for innovation to cycle between the Computer and Social Sciences. Commercial mobile phones effectively function as sensing platforms, effortlessly creating massive datasets. Machine learning and data mining provide powerful analytic lenses to observe and measure people in ways previously not possible.

Examining data describing how routines support everyday activities, we can uncover new subjects for sensing and machine learning. I demonstrate the value of this approach using dual-income families. My studies of family logistics show that unexpected changes to plans cause parents the most anxiety. My data collection using mobile phones shows that that less than $20 \%$ of all days involve no unexpected changes (see Fig. 1). Interview studies show that family members sometimes need but do not have access to information about the plans and routines of other family members. Because family members do not document this information, they do not exist as resources family members can turn to when needed.

These observations create opportunities for Computer Science research that serves people, creating end-user applications to support daily life. Machine learning and data mining can automatically document family logistics with only mobile phone GPS. I create Algorithms that sense when parents pick up and drop children off at over $90 \%$, predict which parent will drive for the next event at over $85 \%$, and predict if a parent will leave a child awaiting their arrival at an activity.

In real-time, we can use predictive models to enable new classes of application. For example, the Person-Place-Time View (PPTV) aggregates our learned models of family coordination into a visualization that promotes person and place to first-class objects (see Fig. 2). In place of the disconnected events you find on calendars, the PPTV shows where everyone is and will be. The PPTV also shows which parent 


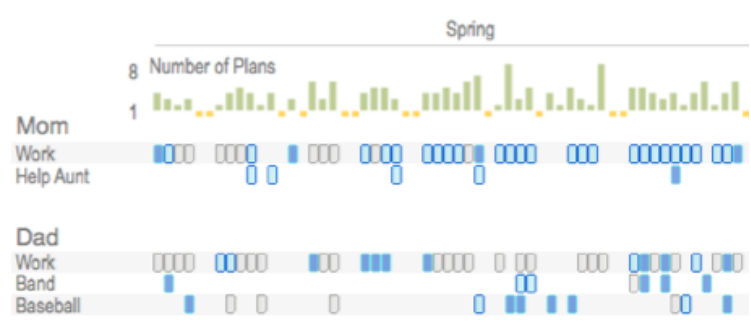

Fig. 1. Family E's activities. Each dot shows an activity instance, (time on x-axis) classified as routine, scheduled or unscheduled deviation. The number of plans created that day, top, shows days with no plan changes in orange, and days with changes in green.

will pick-up and drop-off children at each event, more clearly identifying each parents' responsibilities.

This new class of applications faces unexpected challenges. Translating human values into systems that serve people is not a straight line. When carpool plans break down, I have observed parents anxiously phoning friends, frantic to make new plans. Simulate a system to re-plan carpools, however, and parents feel replaced, violated, and out of control. In Ubicomp systems, functional needs interact with social

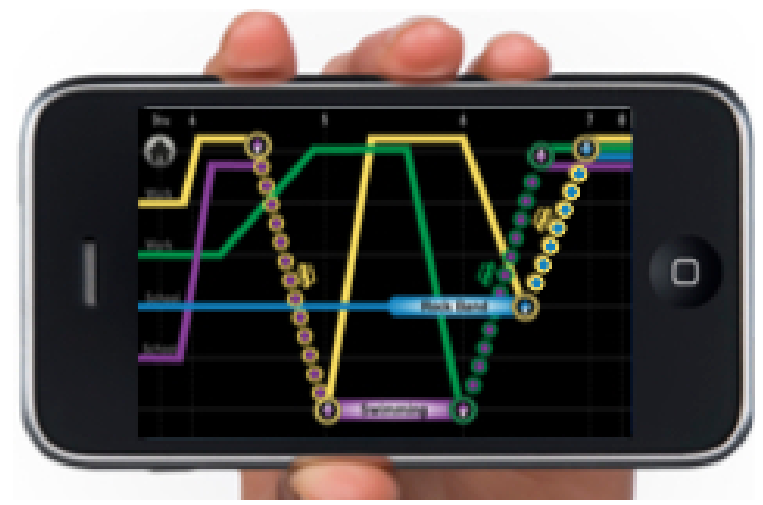

Fig. 2. The PPTV showing a simple plan. Rows are places, colored lines people. Lines are horizontal when a person is at a place, diagonal when traveling. Arrows indicate pick-ups and drop-offs, and dotted lines indicate parent-child co-travel.

values. Deployed systems risk bumping against these unexpected interactions. To avoid this kind of failure, I developed a technique called Speed Dating (SD). SD offers a way to simulate how users experience not only how applications function but how they interact with social values. 